\title{
Etude du dopage de l'arséniure de gallium par la technique d'épitaxie en phase vapeur aux organométalliques
}

\author{
G. Keil, M. Le Métayer, A. Cuquel et D. Le Pollotec \\ Centre National d'Etudes Spatiales, 18, avenue Edouard Belin, 31055 Toulouse Cedex, France
}

(Reçu le 25 janvier 1982, révisé le 5 avril 1982, accepté le 14 avril 1982)

\begin{abstract}
Résumé. - Des couches d'arséniure de gallium épitaxiées par la technique de croissance aux organométalliques ont été dopées par le germanium, l'étain, le sélénium et le zinc. L'étude de l'incorporation de ces éléments en fonction des paramètres de croissance nous permet d'ajuster le niveau de dopage entre $5 \times 10^{16}$ et $3 \times 10^{18} \mathrm{~cm}^{-3}$ tant en type $n$ qu'en type p. Les valeurs de mobilité obtenues dans cette gamme $\left(\mu_{n}\right.$ compris entre 2700 et $3900 \mathrm{~cm}^{2} \cdot \mathrm{V}^{-1} \cdot \mathrm{s}^{-1}$ et $\mu_{\mathrm{p}}$ compris entre 105 et $320 \mathrm{~cm}^{2} \cdot \mathrm{V}^{-1} \cdot \mathrm{s}^{-1}$ ) attestent que les matériaux élaborés présentent une bonne qualité électronique.

Abstract. - The doping of gallium arsenide layers grown by the trimethylgallium/arsine method has been studied for germanium, tin, selenium and zinc doping. We obtained $n$ and $p$ type layers doped between $5 \times 10^{16}$ and $3 \times 10^{18} \mathrm{~cm}^{-3}$. In the growth conditions described, the values of Hall mobilities $\mu_{\mathrm{n}}$ measured are between 2700 and $3900 \mathrm{~cm}^{2} \cdot \mathrm{V}^{-1} \cdot \mathrm{s}^{-1}$ and $\mu_{\mathrm{p}}$ between 105 and $320 \mathrm{~cm}^{2} \cdot \mathrm{V}^{-1} \cdot \mathrm{s}^{-1}$ and are in good agreement with those obtained by the other techniques.
\end{abstract}

1. Introduction. - La croissance épitaxiale par la méthode aux organométalliques développée à l'origine par H. M. Manasevit en 1968 [1] a fait l'objet de nombreuses études. Des dispositifs qui présentent des performances électriques satisfaisantes peuvent être réalisés par cette technique tant dans le domaine des diodes lasers [2] que des composants hyperfréquence [3] et des structures photovoltaïques [4], [5], [6].

Les couches d'arséniure de gallium sont obtenues par pyrolyse du triméthylgallium et de l'arsine. Le gaz vecteur, l'hydrogène, amène les composés dans le réacteur où ils se mélangent; la réaction est localisée au voisinage immédiat du substrat qui seul est chauffé à la température de la réaction de décomposition. Ce procédé dit à « murs froids » diminue les réactions parasites avec les parois du tube de quartz. En travaillant sous basse pression d'hydrogène, suivant la méthode développée par J. P. Duchemin [7], on réduit l'autodopage des couches épitaxiées.

Dans l'objectif de réaliser des structures photovoltaïques d'arséniure de gallium, en vue d'applications spatiales, il paraissait intéressant d'étudier cette technique de croissance, notamment l'incorporation des différents dopants et de comparer les propriétés électriques des couches épitaxiées. On verra que les transitions de dopage obtenues montrent

\footnotetext{
REVUE PHYSIQUE APPLIQUÉE. - T. 17, № 7, JUILLET 1982
}

qu'une telle technique est adaptée à l'élaboration d'homojonction d'arséniure de gallium.

2. Dispositif et conditions expérimentales. - Le réacteur est du type horizontal (Fig. 1). C'est un tube de quartz contenant un suscepteur de graphite passivé par une couche de carbure de silicium légèrement incliné pour améliorer l'uniformité de la croissance. Ce dernier est chauffé par induction haute fréquence.

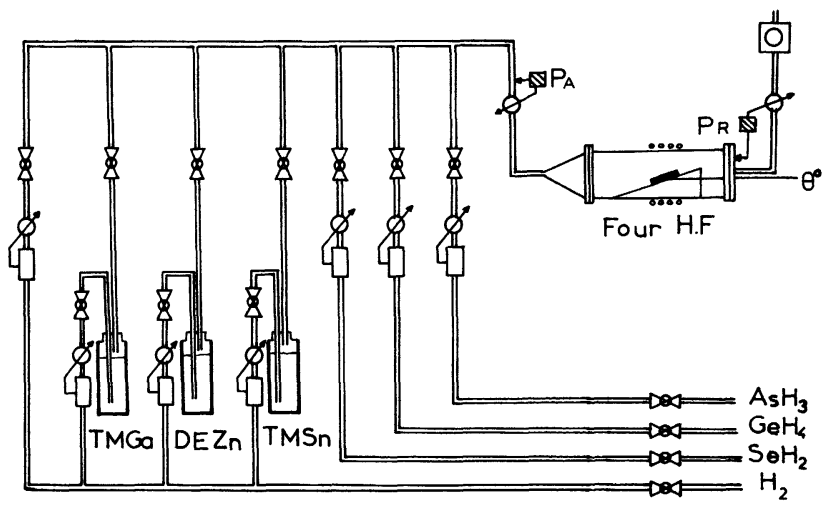

Fig. 1. - Schéma de l'installation d'épitaxie.

[Schematic of chemical vapour deposition apparatus.] 
La température des échantillons est mesurée à l'aide d'un thermocouple chromel-alumel disposé dans le suscepteur. La pression à l'intérieur du réacteur peut être réglée entre quelques torrs et la pression atmosphérique à l'aide d'une vanne d'étranglement couplée à une pompe primaire. Une autre vanne, placée en amont du réacteur, maintient une pression égale à la pression atmosphérique à l'admission des gaz. Les différents débits sont contrôlés par des débitmètres massiques. Les composés organométalliques sont contenus dans des barboteurs thermostatés, et sont traversés par un flux d'hydrogène.

On utilise de l'arsine $\mathrm{AsH}_{3}$ diluée à $10 \%$ dans l'hydrogène et le triméthylgallium est thermostaté à $-5^{\circ} \mathrm{C}$

Les gaz dopants utilisés sont : gène,

- le germane $\left(\mathrm{GeH}_{4}\right)$ dilué à $25 \mathrm{ppm}$ dans l'hydro-

- l'hydrogène sélénié $\left(\mathrm{SeH}_{2}\right)$ dilué à $100 \mathrm{ppm}$ dans l'hydrogène,

- le tétraméthylétain thermostaté à $-15^{\circ} \mathrm{C}$,

- le diéthylzinc thermostaté à $-15^{\circ} \mathrm{C}$.

Les substrats d'arséniure de gallium sur lesquels sont effectués les dépôts sont désorientés de $3^{\circ}$ par rapport au plan (001) et possèdent une face polie optique. Ils subissent une opération de dégraissage suivie d'un décapage chimique avec une solution $\mathrm{H}_{2} \mathrm{SO}_{4}, \mathrm{H}_{2} \mathrm{O}_{2}, \mathrm{H}_{2} \mathrm{O}$ dans les proportions 5.1 .1 avant d'être introduits dans le réacteur.

Après une mise sous vide, on établit un débit d'hydrogène et une pression dans le réacteur qui dans toute cette étude ont été maintenus respectivement à $10 \mathrm{l} / \mathrm{min}$. et 76 torrs. Le substrat est ensuite porté à la température de croissance sous un balayage d'arsine afin d'éviter toute décomposition de l'arséniure de gallium. On établit alors les débits de triméthylgallium et de dopant pour réaliser la croissance.

L'étude paramétrique de la vitesse de croissance a été effectuée en fonction de la fraction molaire de triméthylgallium, de la température de dépôt, de la pression partielle d'arsine et de la position du substrat le long du suscepteur. Une étude thermodynamique [8] a abouti à une bonne corrélation avec les résultats expérimentaux.

On obtient une vitesse de croissance indépendante de la fraction molaire d'arsine et de la température du dépôt dans la gamme 500 à $750^{\circ} \mathrm{C}$. Par contre, une loi de variation linéaire relie la vitesse de croissance à la fraction molaire de triméthylgallium (TMG).

Ces résultats confirment les travaux antérieurs de J. P. Duchemin [7], de J. P. Hallais [9] et J. S. Bass [10].

L'étude du dopage résiduel démontre que le facteur principal agissant sur le niveau de dopage est le rapport As/Ga comme l'ont constaté J. S. Bass [10] et Y. Seki [11]. On observe dans nos conditions expérimentales un changement rapide du type de conduction pour une valeur critique du rapport $\mathrm{As} / \mathrm{Ga}$ égale à 7 (Fig. 2).

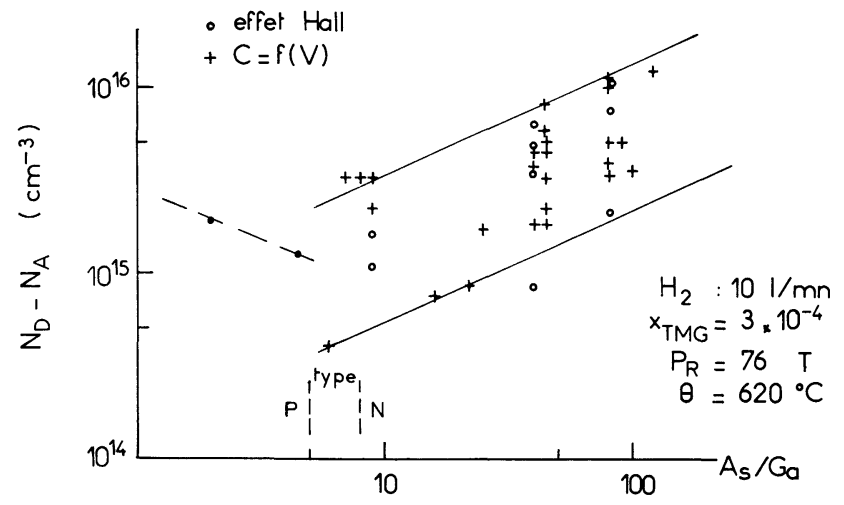

Fig. 2. - Influence du rapport As/Ga sur le dopage résiduel.

[Residual doping as a function of the As/Ga ratio.]

Pour l'étude du dopage intentionnel, nous avons choisi un rapport $\mathrm{As} / \mathrm{Ga}$ égal à 40 . Ce rapport est celui qui permet d'obtenir un dopage résiduel de l'ordre de $1,5 \times 10^{15} \mathrm{~cm}^{-3}$ avec des valeurs de mobilité de Hall égales à $6280 \mathrm{~cm}^{2} \cdot \mathrm{V}^{-1} \cdot \mathrm{s}^{-1}$ à $300 \mathrm{~K}$ et $28500 \mathrm{~cm}^{2} \cdot \mathrm{V}^{-1} \cdot \mathrm{s}^{-1}$ à $77 \mathrm{~K}$.

3. Dopage des couches épitaxiées. - Le dopage volontaire des couches au cours d'une épitaxie est effectué par l'introduction dans la phase vapeur d'une quantité contrôlée de l'élément dopant.

L'étude du dopage de type $\mathrm{n}$ porte sur deux sortes de dopant :

- des éléments IV de la classification périodique qui sont donneurs sur site gallium : le germanium et l'étain;

- un élément VI qui est donneur sur site arsenic : le sélénium.

Le dopage de type $\mathrm{p}$ de l'arséniure de gallium est obtenu par l'élément le plus communément utilisé : le zinc. C'est un élément II de la classification périodique qui est accepteur.

Pour chacun de ces dopants, on a étudié expérimentalement l'influence des différentes fractions molaires (dopant, arsine, triméthylgallium) et de la température sur la concentration de porteurs libres des couches épitaxiées. La valeur du niveau de dopage est obtenue par mesure capacitive à l'aide d'un profileur électrochimique [12].

3.1 Dopage PAR LE GERMANIUM. - Avec un mélange de gaz de $25 \mathrm{ppm}$ de germane dans l'hydrogène et avec les conditions expérimentales citées au paragraphe précédent, il est possible de faire varier la fraction molaire de germane dans le réacteur entre $5 \times 10^{-9}$ et $6 \times 10^{-7}$. Dans cette gamme, la concentration de porteurs libres est une fonction croissante de la fraction molaire de germane quand tous les autres paramètres de croissance sont maintenus constants (Fig. 3). 


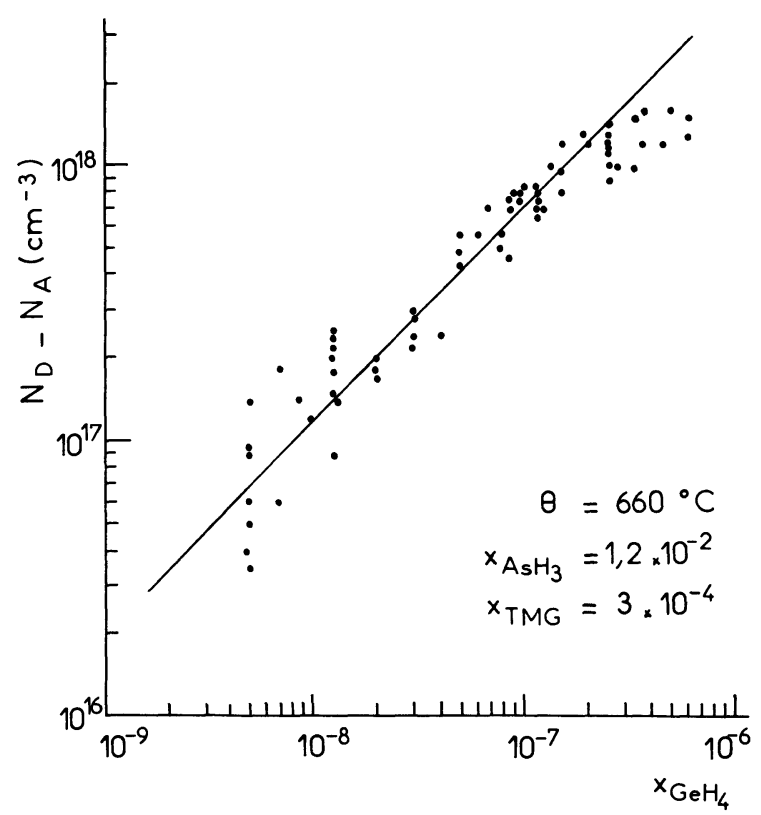

Fig. 3. - Dopage germanium : Variation de la concentration de porteurs avec la fraction molaire de germane.

[Germanium doping : Electron concentration as a function of germane concentration.]

Pour une concentration donnée de germane dans la phase vapeur, le niveau de dopage des couches épitaxiées présente une légère décroissance avec une augmentation de la pression partielle d'arsine comme cela est illustré par la figure 4 .

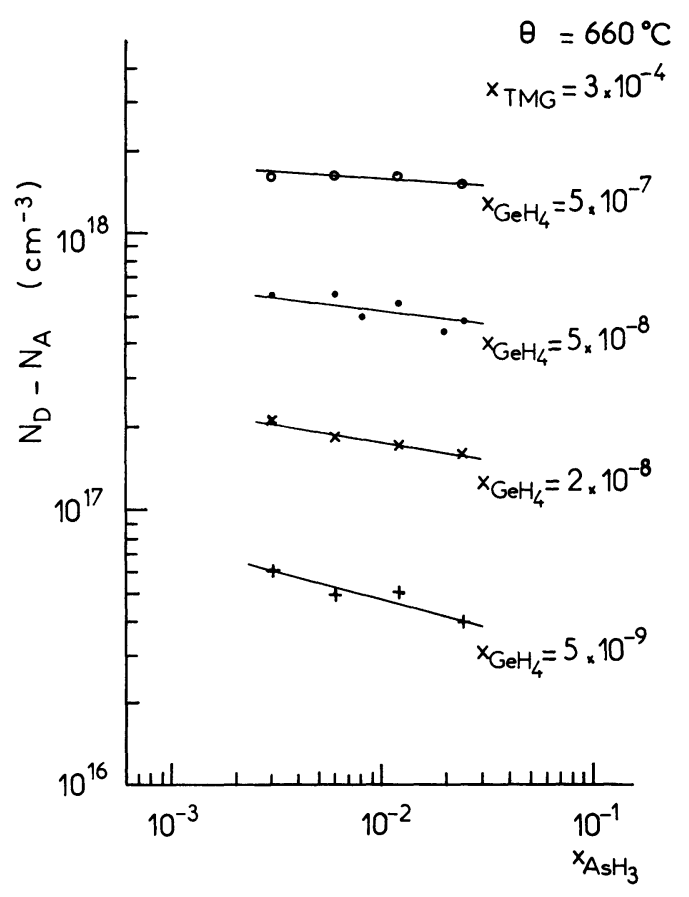

Fig. 4. - Dopage germanium : Variation de la concentration de porteurs avec la fraction molaire d'arsine.

[Germanium doping : Electron concentration as a function of arsine concentration.]
La figure 5 montre la loi de variation reliant la vitesse de croissance au niveau de porteurs libres quand tous les autres paramètres sont maintenus constants. On observe que lorsque la vitesse de croissance augmente, le nombre d'impuretés contenues dans la couche épitaxiée diminue. L'incorporation du germanium est donc régie par le rapport du flux de germanium et de gallium à l'interface substrat-vapeur.

Enfin, lorsque tous les paramètres de croissance sont figés, excepté la température du dépôt, le niveau de dopage s'accroît exponentiellement avec la température (Fig. 6) signifiant une activation thermique de la réaction de décomposition.

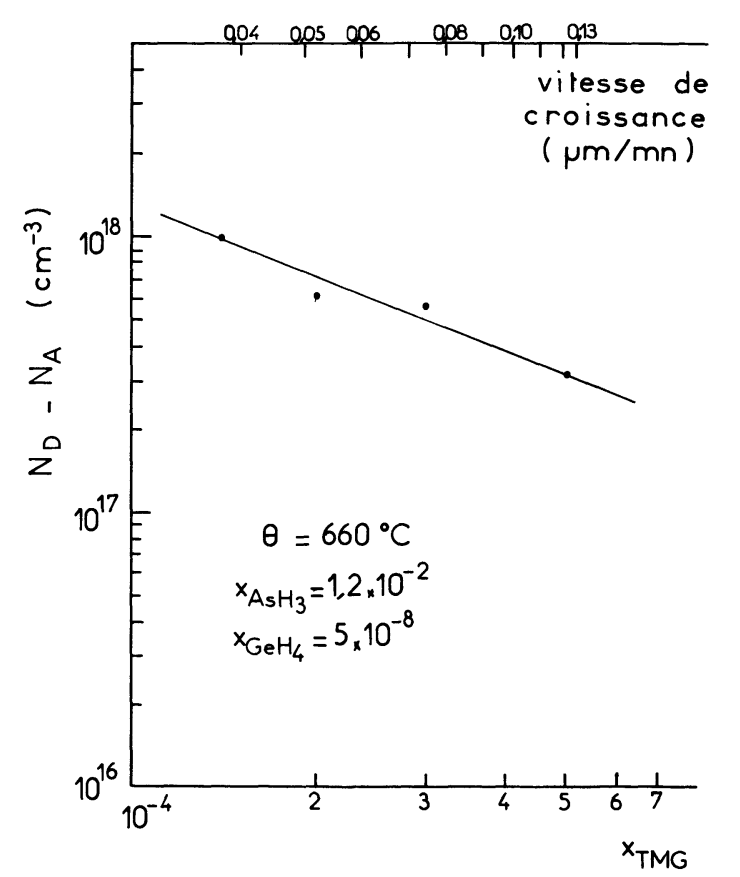

Fig. 5. - Dopage germanium : Variation de la concentration de porteurs avec la fraction molaire de triméthylgallium.

[Germanium doping : Electron concentration as a function of trimethylgallium concentration.]

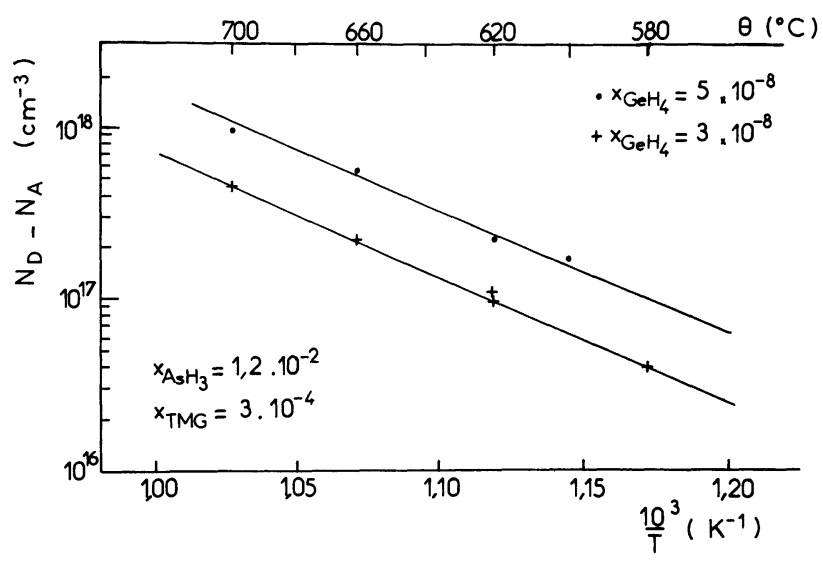

Fig. 6. - Dopage germanium : Variation de la concentration de porteurs avec la température.

[Germanium doping : Electron concentration as a function of temperature.] 
J. P. Duchemin et al. [7] qui avaient étudié partiellement le dopage de l'arséniure de gallium par le germanium avaient conclu à un mécanisme d'incorporation similaire entre le silicium et le germanium. Les résultats obtenus semblent indiquer que l'incorporation du germanium dans l'arséniure de gallium répond à un mécanisme sensiblement différent de celui du silicium dans le même matériau. On peut dire que l'incorporation du germanium est contrôlée par la cinétique de croissance et limitée par la décomposition du germane et la fraction molaire d'arsine.

Nous avons étudié, par ailleurs, l'élimination du gaz dopant de la phase vapeur afin de déterminer les meilleures conditions d'élaboration de couches successives possédant des concentrations en impuretés très différentes. La figure 7 montre une structure composée de deux couches dopées au germanium : une fortement dopée (couche tampon) puis une couche de niveau de dopage plus faible. Le tracé du profil est effectué par le profileur électrochimique.

On voit ainsi qu'il est possible d'obtenir des changements brusques du niveau de dopage au cours d'une même épitaxie.

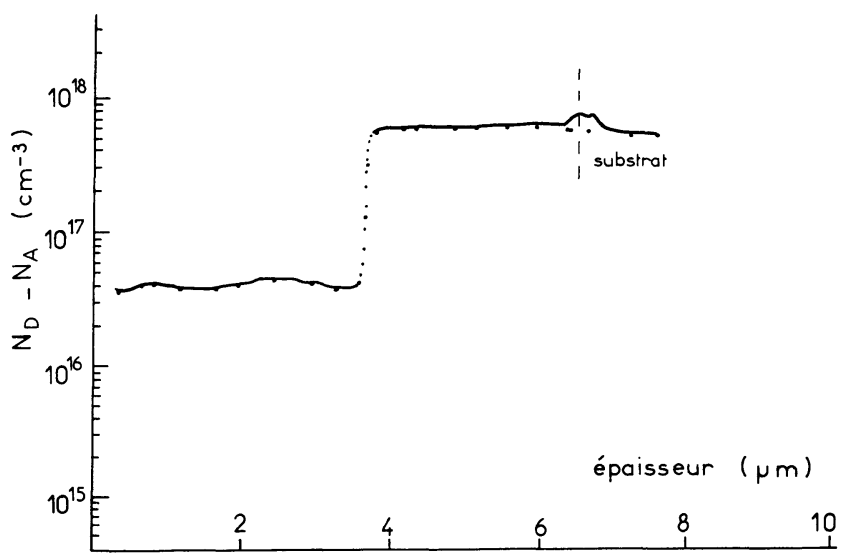

Fig. 7. - Profil de dopage au germanium.

[Impurity profile of germanium doping layer.]

3.2 Dopage PAR L'ÉtAin. - L'étain est introduit dans le réacteur sous forme d'organométallique : le tétraméthylétain (TMSn). La fraction molaire de ce composé est contrôlée par le débit d'hydrogène barbotant dans le liquide et la température du bain thermostaté.

Dans l'arséniure de gallium, l'étain est comme le germanium une impureté à caractère amphotère. On pourrait s'attendre à trouver des lois de variations similaires du niveau de dopage en fonction des paramètres de croissance que celles obtenues par l'étude du dopage par le germanium.

Les figures 8,9 et 10 qui illustrent respectivement l'influence de la température, de la vitesse de croissance et de la fraction molaire de tétraméthylétain sur la concentration d'impuretés incorporées, mon-

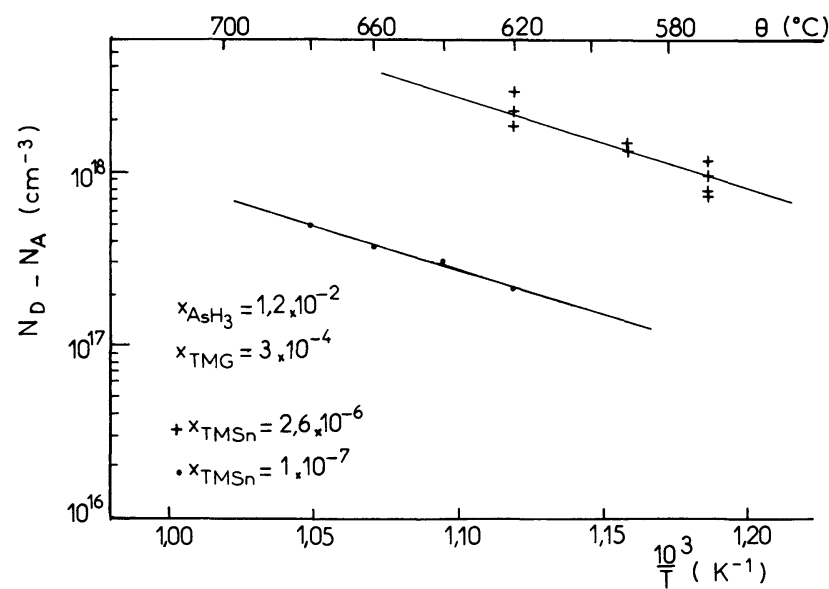

Fig. 8. - Dopage étain : Variation de la concentration de porteurs avec la température.

[Tin doping : Electron concentration as a function of temperature.]

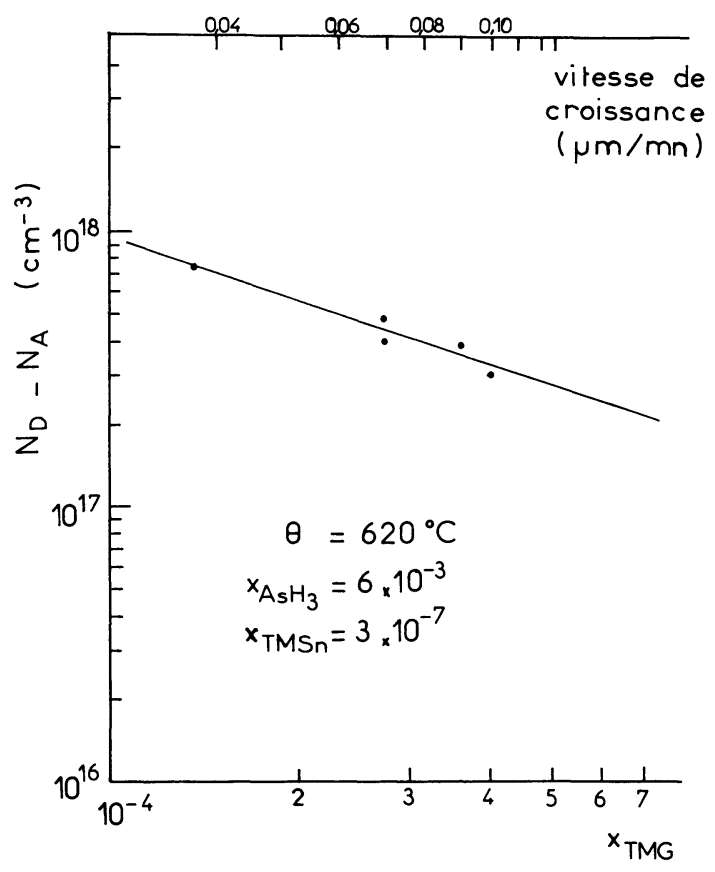

Fig. 9. - Dopage étain : Variation de la concentration de porteurs avec la fraction molaire de triméthylgallium.

[Tin doping : Electron concentration as a function of trimethylgallium concentration.]

trent effectivement une incorporation de l'étain comparable à celle du germanium. La dispersion des mesures aux faibles valeurs de $x_{\text {TMSn }}$ (Fig. 10) est imputable à l'emploi de très faibles débits dont le contrôle est délicat à maîtriser.

Cependant, quand on augmente la quantité d'arsine présente dans la phase vapeur, tous les autres paramètres de croissance étant maintenus constants, on augmente le nombre de lacunes de gallium à la surface de réaction. Comme le degré de préférence de l'étain 


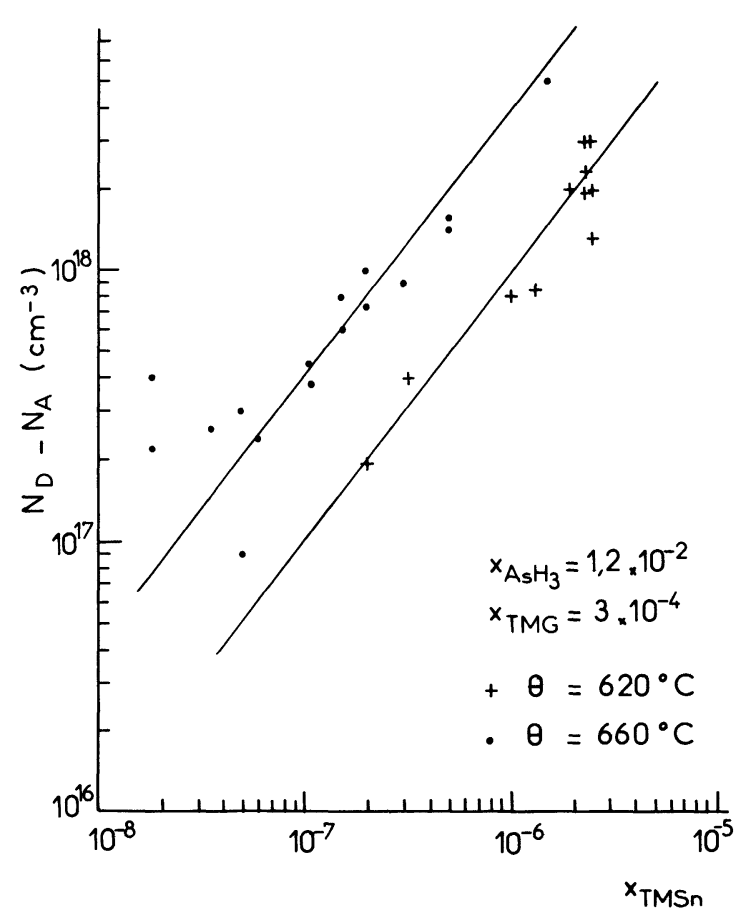

Fig. 10. - Dopage étain : Variation de la concentration de porteurs avec la fraction molaire de tétraméthylétain.

[Tin doping : Electron concentration as a function of tetramethyltin concentration.]

pour un site $\mathrm{Ga}$, ou pour un site As, dépend de l'environnement de croissance, on favorise ainsi l'incorporation de $\mathrm{Sn}$ sur site $\mathrm{Ga}$, ce qui conduit à une augmentation du nombre de porteurs libres comme le rapporte la figure 11 .

L'incorporation de l'étain semble donc être un phénomène dominé par la cinétique de croissance.

3.3 DOPAGe PAR Le sélénium. - On utilise le sélénium sous la forme d'hydrure $\mathrm{SeH}_{2}$ (hydrogène sélénié) dilué à $100 \mathrm{ppm}$ dans l'hydrogène.

Les séries d'expériences entreprises afin de déterminer l'influence des différents paramètres sur le nombre d'impuretés incorporées nous font observer les phénomènes suivants.

Il existe une relation linéaire entre la fraction molaire $\mathrm{de} \mathrm{SeH}_{2}$ et le niveau de dopage comme cela est montré sur la figure 12. Notons que pour un dopage équivalent de l'arséniure de gallium, il faut introduire 10 fois plus de dopant dans la phase vapeur lorsqu'on remplace le germane par l'hydrogène sélénié toutes choses étant égales par ailleurs.

Les figures 13 et 14 indiquent respectivement que la concentration d'impuretés incorporées dans la couche épitaxiée est inversement proportionnelle à la fraction molaire d'arsine et croît avec la vitesse de croissance. On peut donc considérer que l'incorporation du sélénium obéit aux équations :

et

$$
\mathrm{SeH}_{2} \rightarrow \mathrm{Se} \text { (vapeur) }+\mathrm{H}_{2}
$$

$$
\mathrm{Se} \text { (vapeur) + lacune } \mathrm{As} \rightleftarrows \mathrm{Se} \text { (site As) }
$$

parce qu'une augmentation du nombre de lacunes d'arsenic à la surface de réaction provient soit d'une phase vapeur appauvrie en arsine, soit d'une concentration de triméthylgallium plus élevée.

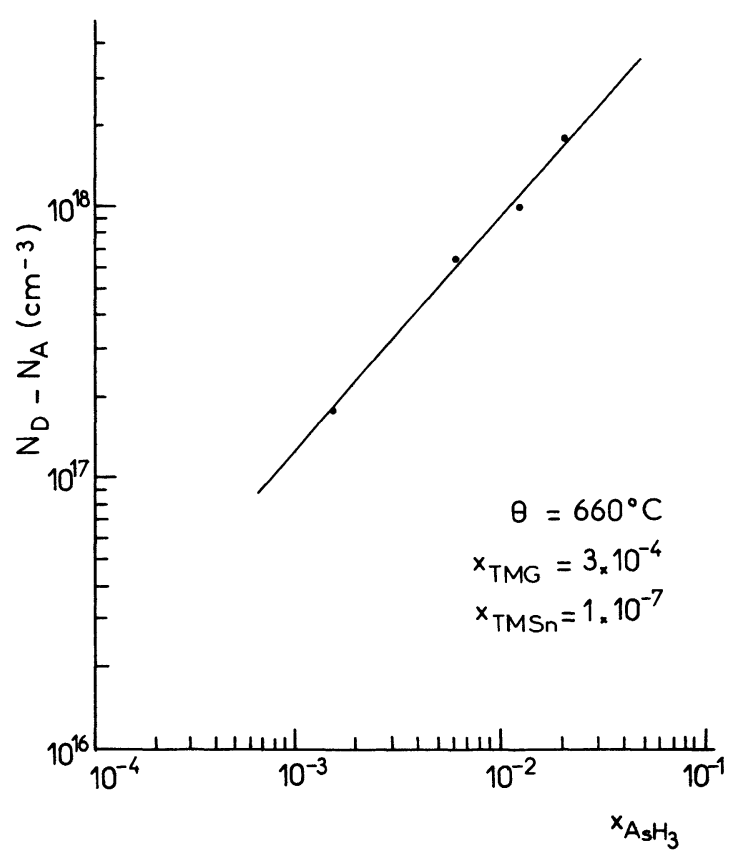

Fig. 11. - Dopage étain : Variation de la concentration de porteurs avec la fraction molaire d'arsine.

[Tin doping : Electron concentration as a function of arsine concentration.]

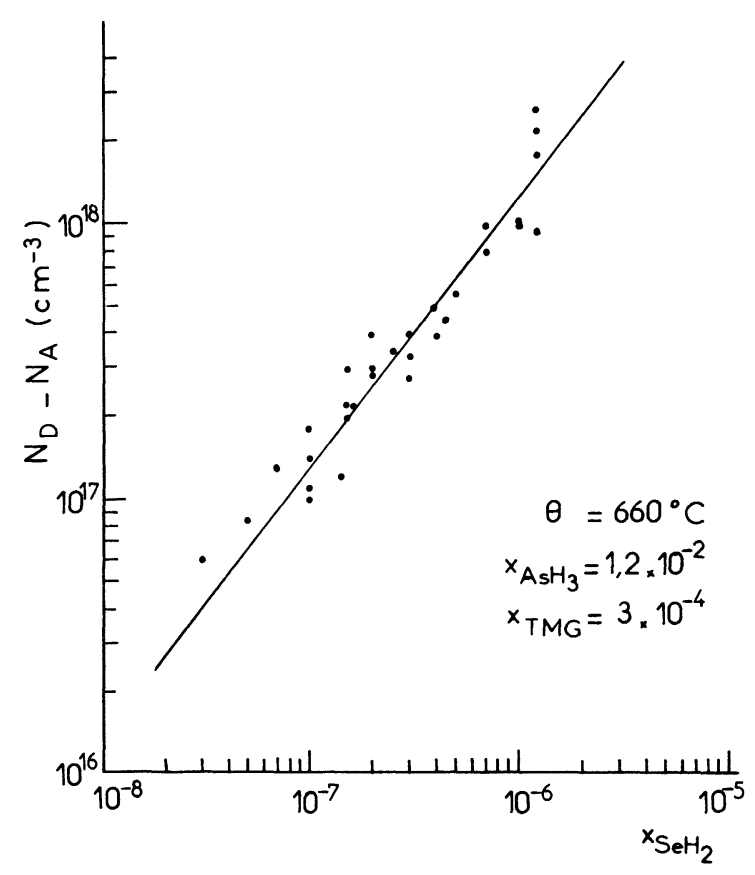

Fig. 12. - Dopage sélénium : Variation de la concentration de porteurs avec la fraction molaire d'hydrogène sélénié.

[Selenium doping : Electron concentration as a function of hydrogen selenide concentration.] 


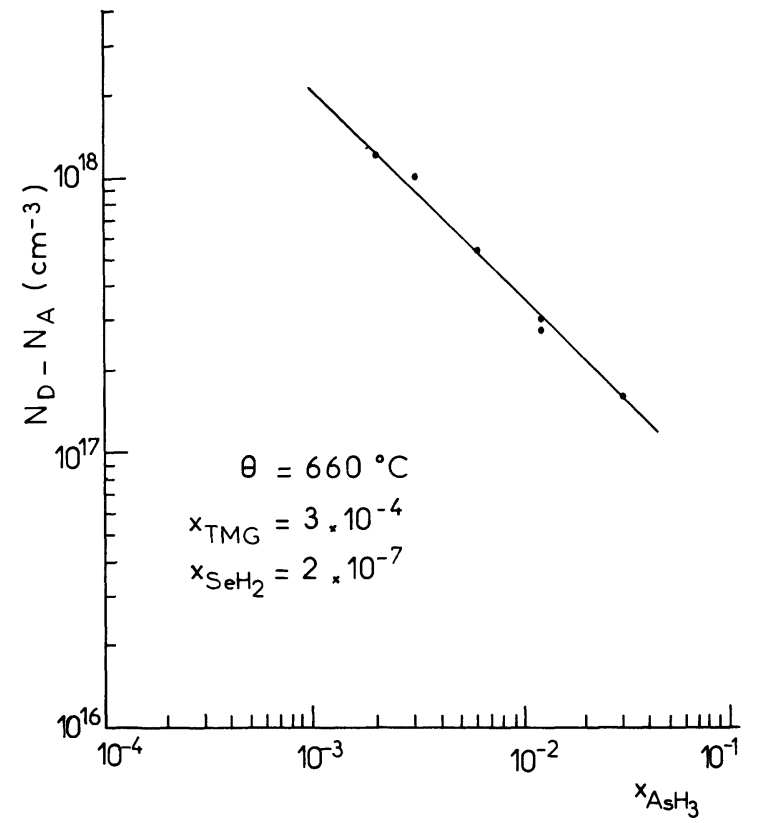

Fig. 13. - Dopage sélénium : Variation de la concentration de porteurs avec la fraction molaire d'arsine.

[Selenium doping : Electron concentration as a function of arsine concentration.]

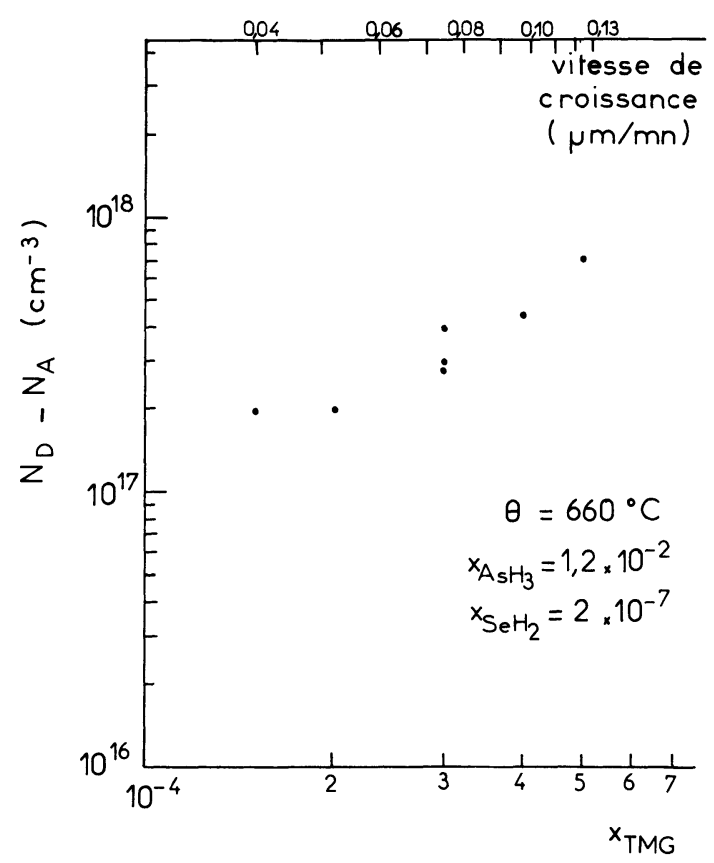

Fig. 14. - Dopage sélénium : Variation de la concentration de porteurs avec la fraction molaire de triméthylgallium.

[Selenium doping : Electron concentration as a function of trimethylgallium concentration.]

L'influence de la température de croissance sur le niveau de dopage, quand tous les autres paramètres de croissance sont maintenus constants, est donnée par la figure 15. Cette courbe laisse supposer une incorporation par adsorption du sélénium qui, dans

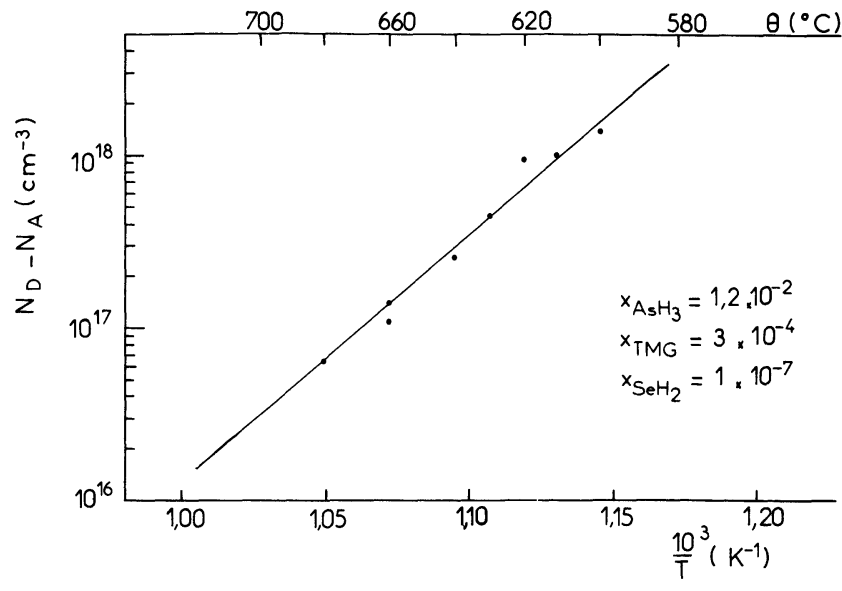

Fig. 15. - Dopage sélénium : Variation de la concentration de porteurs avec la température.

[Selenium doping : Electron concentration as a function of temperature.]

les conditions de température et de pression citées, se trouve à l'état gazeux à l'interface avec le substrat (Eq. 1).

Lorsque la température croit, l'incorporation du sélénium sur site arsenic devient alors plus difficile et on observe un niveau de dopage qui est une fonction inverse de la température de croissance.

Ces résultats confirment les travaux antérieurs de J. P. Hallais [9] et montreraient une incorporation de l'impureté sélénium sur site arsenic.

La figure 16 illustre une transition de dopage entre deux niveaux très différents. Il s'agit d'une structure $\mathrm{n} / \mathrm{n}^{+}$composée d'une couche dopée au sélénium $\left(4 \times 10^{17} \mathrm{~cm}^{-3}\right)$ suivie d'une couche non intentionnellement dopée, déposée sur substrat fortement dopé au silicium. Rappelons que les impuretés qui sont à l'origine du dopage résiduel proviennent des réactifs initiaux $\left(\mathrm{AsH}_{3}, \mathrm{TMG}\right.$, gaz vecteur).

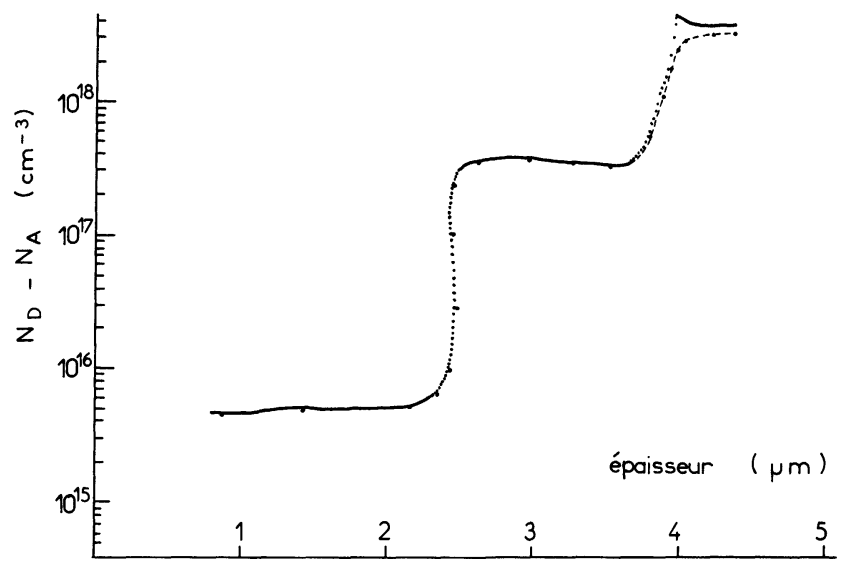

Fig. 16. - Profil d'impuretés entre un dopage au sélénium et un dopage résiduel.

[Impurity profile of residual doping-selenium doping layer.] 
La transition abrupte entre ces deux niveaux de dopage indique une bonne élimination de l'hydrogène sélénié de la phase vapeur.

3.4 DOPAGE PAR LE ZINC. - L'incorporation de l'élément zinc dans l'arséniure de gallium donne une conduction de type p. Le dopage des couches est obtenu en introduisant dans la phase vapeur du diéthylzinc (DEZ). Ce composé organométallique thermostaté à $-15^{\circ} \mathrm{C}$ est entraîné par l'hydrogène vers le réacteur.

Le taux de concentration des couches dopées par le zinc peut être ajusté entre $7 \times 10^{16}$ et $4 \times 10^{18} \mathrm{~cm}^{-3}$. La figure 17 montre la relation linéaire liant le niveau de dopage à la fraction molaire de diéthylzinc.

La variation du nombre d'impuretés incorporées en fonction de la concentration d'arsine présente dans la phase vapeur est donnée par la figure 18. Le niveau de porteurs s'accroît avec une augmentation de la fraction molaire d'arsine.

La figure 19 montre l'influence de la température sur le niveau de dopage quand tous les autres paramètres sont maintenus constants.

Ces résultats sont comparables à ceux obtenus par S. J. Bass [14] bien qu'il utilise le diméthylzinc (DMZ).

Par contre, les mesures effectuées sur des couches élaborées à des vitesses de croissance différentes indiquent que le niveau de porteurs est indépendant de la quantité de triméthylgallium introduite dans la phase vapeur (Fig. 20). L'incorporation du zinc ne semble donc pas être contrôlée par la cinétique de croissance.

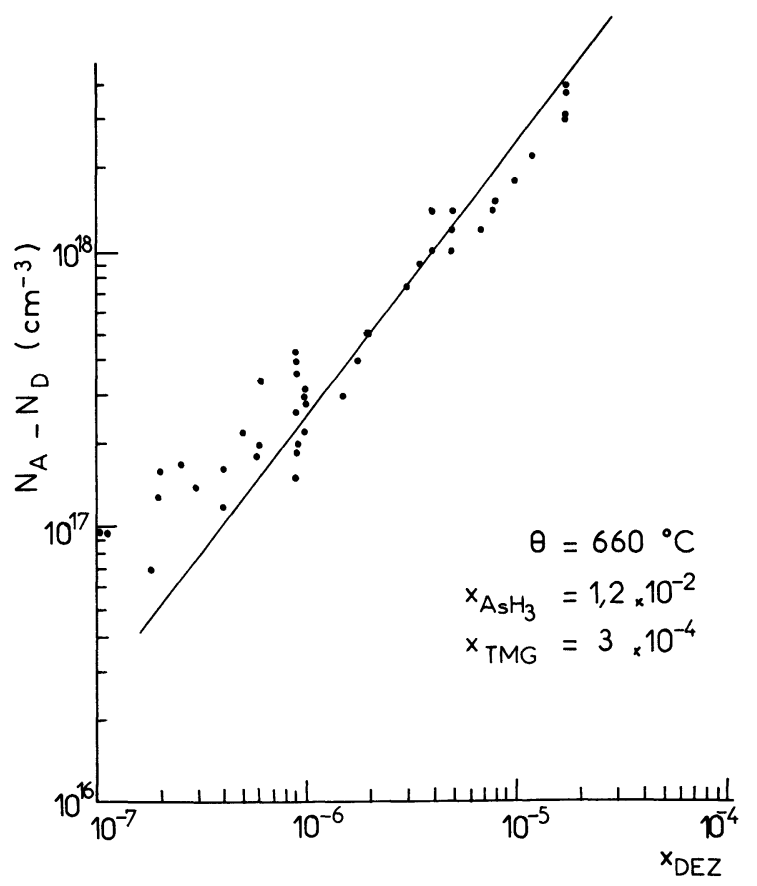

Fig. 17. - Dopage zinc : Variation de la concentration de porteurs avec la fraction molaire de diéthylzinc.

[Zinc doping : Hole concentration as a function of diethylzinc concentration.]

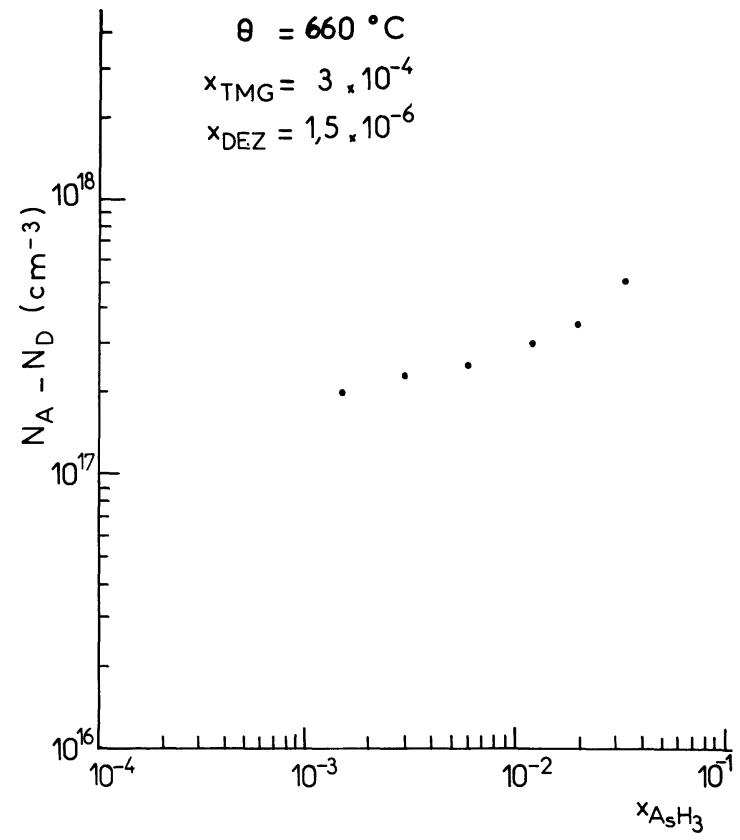

Fig. 18. - Dopage zinc : Variation de la concentration de porteurs avec la fraction molaire d'arsine.

[Zinc doping : Hole concentration as a function of arsine concentration.]

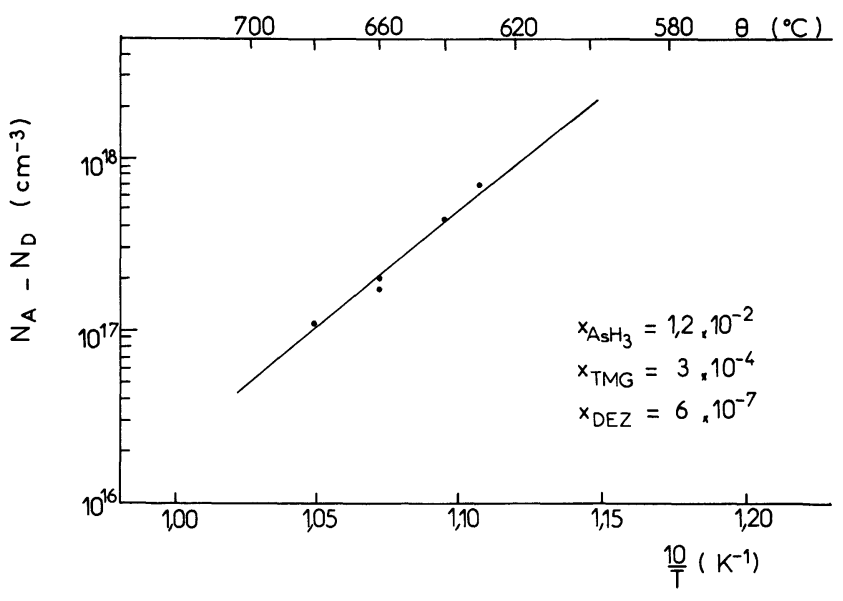

Fig. 19. - Dopage zinc : Variation de la concentration de porteurs avec la température.

[Zinc doping : Hole concentration as a function of temperature.]

Les transitions de dopage obtenues sur des structures $\mathrm{p} / \mathrm{p}^{+}$présentent des profils aussi abrupts que ceux observés sur des structures $n / n^{+}$. La figure 21 fait apparaître une structure composée d'une couche $\mathrm{p}^{+}$épitaxiée avec une fraction molaire de diéthylzinc égale à $1 \times 10^{-5}$ suivie d'une couche dopée avec $x_{\mathrm{DEZ}}=4 \times 10^{-7}$, sur substrat fortement dopé au $\operatorname{zinc}\left(2,8 \times 10^{18} \mathrm{~cm}^{-3}\right)$.

L'élimination du diéthylzinc de la phase vapeur ne pose donc pas de problèmes. 


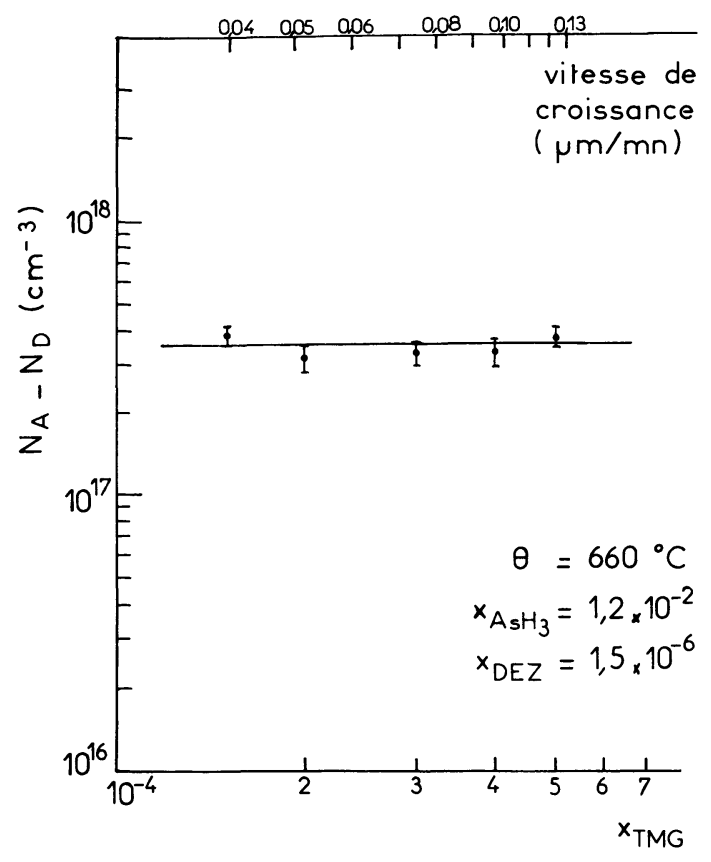

Fig. 20. - Dopage zinc : Variation de la concentration de porteurs avec la fraction molaire de triméthylgallium.

[Zinc doping : Hole concentration as a function of trimethylgallium concentration.]

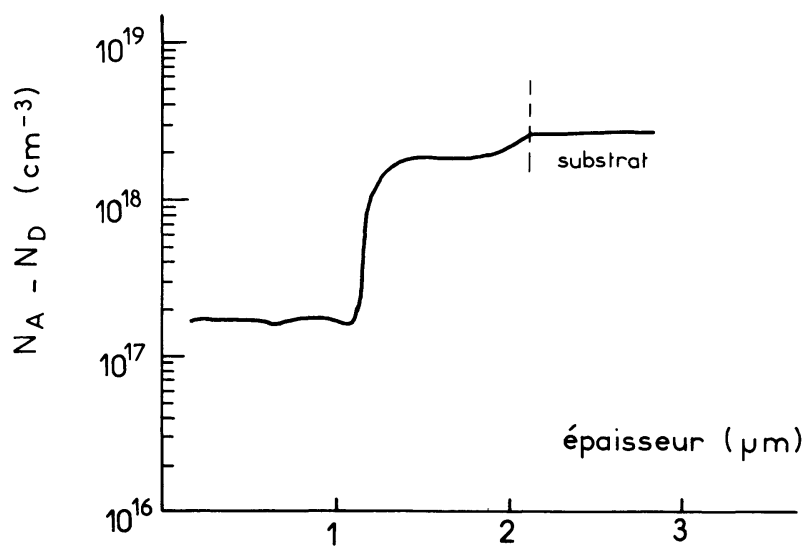

Fig. 21. - Profil de dopage au zinc.

[Impurity profile of zinc doping layer.]

4. Propriétés électriques. - Afin de déterminer la pureté et la qualité de nos dépôts, nous avons étudié des couches d'arséniure de gallium dopées indifféremment au germanium, sélénium, étain et zinc, en mesurant l'une des caractéristiques électriques du matériau : la mobilité des porteurs majoritaires.

Les mesures d'effet Hall, réalisées par la méthode de Van der Pauw, rapportent des valeurs de mobilité de Hall, obtenues à $300 \mathrm{~K}$ très proches des valeurs de référence [15] comme le montrent les figures 22 et 23 ; soulignons que l'emploi du sélénium comme élément dopant conduit, comme nous nous y attendions du

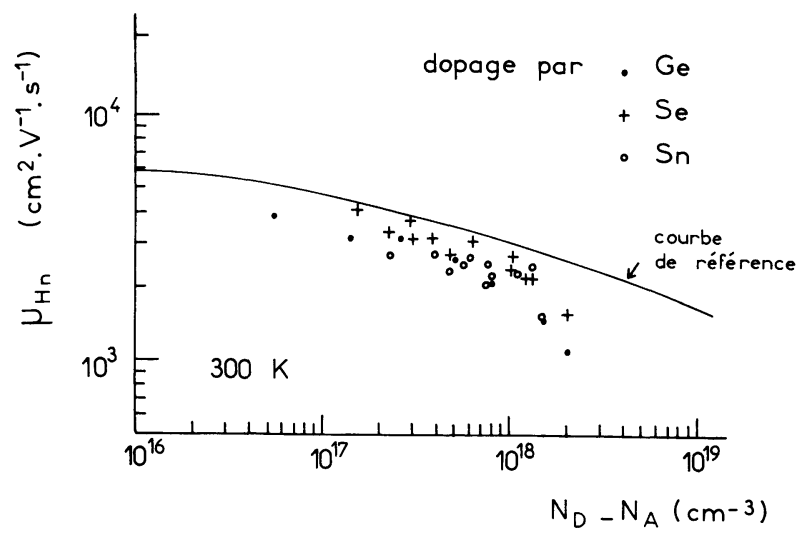

Fig. 22. - Valeurs de mobilité de Hall en fonction de la concentration de porteurs dans l'AsGa de type n à $300 \mathrm{~K}$.

[Hall mobility as a function of carrier concentration in $\mathrm{n}$ type $\mathrm{GaAs}$ layers at $300 \mathrm{~K}$.]

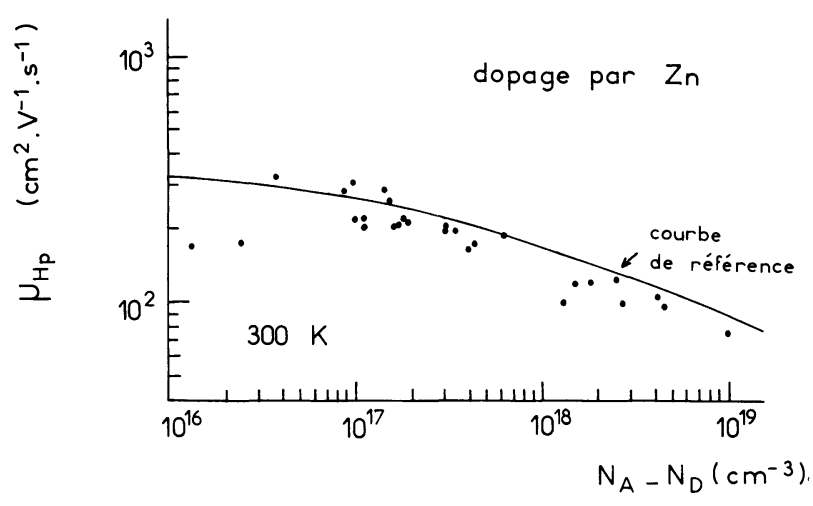

Fig. 23. - Valeurs de mobilité de Hall en fonction de la concentration de porteurs dans l'AsGa de type p à $300 \mathrm{~K}$.

[Hall mobility as a function of carrier concentration in ptype GaAs layers at $300 \mathrm{~K}$.]

fait du caractère non amphotère de cette impureté, à des valeurs de mobilité plus élevées que celles obtenues sur des couches dopées au germanium ou à l'étain.

Toutes les valeurs de mobilité concordent avec les résultats publiés par d'autres laboratoires travaillant sur la technique d'épitaxie en phase vapeur de l'arséniure de gallium ([7], [13], [16).

D'autre part, nous avons cherché à évaluer la longueur de diffusion par la technique des courants induits à l'aide du microscope électronique à balayage.

La méthode de Morton-Hayne [17] consiste à balayer la zone active d'une jonction avec un faisceau électronique et à relever la variation du courant de court-circuit en fonction de la distance de l'injection électronique par rapport à la jonction.

En travaillant à $15 \mathrm{keV}$ sur une jonction $\mathrm{p}^{+} / \mathrm{n}$ dopée respectivement au zinc $\left(3 \times 10^{18} \mathrm{~cm}^{-3}\right)$ en face avant et au sélénium $\left(2,5 \times 10^{17} \mathrm{~cm}^{-3}\right)$ en zone active, on a obtenu une longueur de diffusion des trous de l'ordre de $2 \mu \mathrm{m}$ comme le montre la figure 24 . 


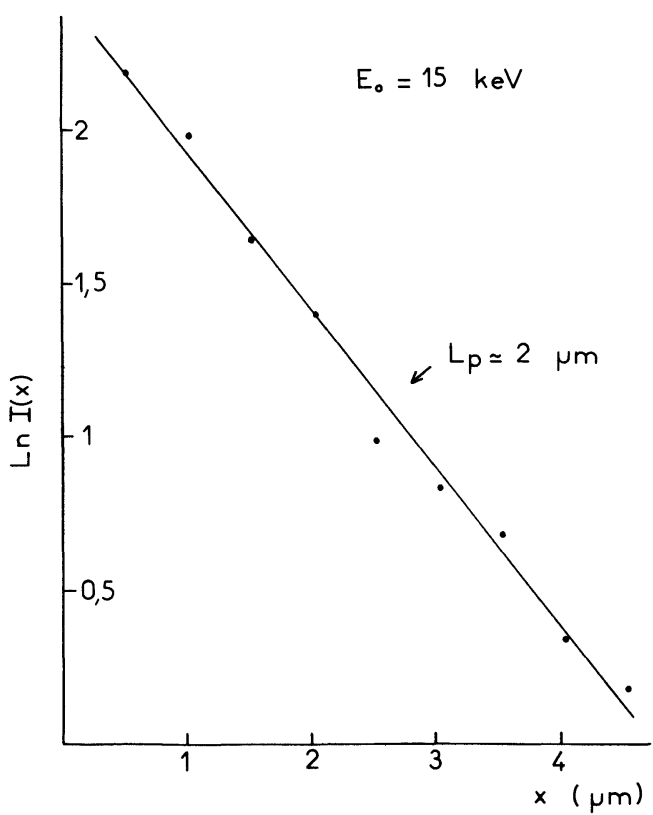

Fig. 24. - Variation du courant de court-circuit avec la distance entre l'injection électronique et la jonction.

[Short circuit current as a function of the distance between the electron injection and the junction.]

5. Conclusion. - Par notre étude, on s'est efforcé de déterminer les particularités d'incorporation des éléments $\mathrm{Ge}, \mathrm{Sn}$, Se et $\mathrm{Zn}$ dans l'arséniure de gallium élaboré par la technique de croissance en phase vapeur aux organométalliques sous basse pression.

Une première interprétation des relations existantes entre les paramètres de croissance et les caractéristiques des couches épitaxiées laisse supposer un mécanisme d'incorporation particulier pour chaque impureté étudiée. Cependant, en comparant les résultats obtenus par les différents dopants pour la même variation d'un paramètre de croissance, on observe que :
- Ge et $\mathrm{Sn}$, ont un coefficient d'incorporation en fonction de la température opposé à celui de $\mathrm{Se}$ et $\mathrm{Zn}$. La thermodynamique des réactions de décomposition des produits utilisés indique que Ge et $\mathrm{Sn}$ se condensent à la surface de réaction tandis que $\mathrm{Se}$ et $\mathrm{Zn}$ restent en phase gazeuse dans nos conditions expérimentales. Il s'ensuit qu'une augmentation de la température, dans le premier cas, favorise l'incorporation des impuretés et rend difficile les échanges à l'interface substratvapeur dans le second cas.

- Les éléments dopants tels que Ge et Se montrent un taux d'incorporation en fonction de la fraction molaire d'arsine inverse à celui des impuretés $\mathrm{Sn}$ et $\mathrm{Zn}$.

- Se présente une incorporation contraire aux impuretés $\mathrm{Ge}$ et $\mathrm{Sn}$ vis-à-vis de la quantité de gallium introduite dans la phase vapeur qui peut être attribuée au fait que $\mathrm{Ge}$ et $\mathrm{Sn}$ se condensent à la surface de réaction. En effet, pour une fraction molaire de $\mathrm{Ge}$ ou Sn donnée et des conditions expérimentales fixées, plus on augmente la vitesse de croissance, moins il y a d'impuretés incorporées dans l'arséniure de gallium ainsi épitaxié.

D'un point de vue électronique, le dopage de l'arséniure de gallium par le sélénium semble conférer au matériau une meilleure mobilité aux électrons $\left(\mu_{\mathrm{H}}=4160 \mathrm{~cm}^{2} \cdot \mathrm{V}^{-1} \cdot \mathrm{s}^{-1}\right.$ pour $\left.n=1,5 \times 10^{17} \mathrm{~cm}^{-3}\right)$.

Enfin, les transitions de dopage entre deux niveaux très différents, qui ont été obtenues, indiquent une bonne élimination des éléments dopants de la phase vapeur. La rapidité avec laquelle s'effectue l'évacuation des impuretés du voisinage immédiat de la zone de réaction est tributaire du coefficient de diffusion des impuretés $\left(D=D_{0} \frac{P_{0}}{P}\right)$ lui-même inversement proportionnel à la pression.

Le procédé d'épitaxie aux organométalliques sous basse pression est donc une méthode attrayante en vue du développement de dispositifs à l'arséniure de gallium.

\section{Bibliographie}

[1] Manasevit, H. M., Appl. Phys. Lett. 12 (1968) 156.

[2] Dupuis, R. D., J. Crystal Growth 55 (1981) 213.

[3] Nakanisi, T., Udagawa, T., Tanaka, A. and Kamei, K., J. Crystal Growth 55 (1981) 255.

[4] DuPuIs, R. D. et al., 14th IEEE PVSC (1980) 1388.

[5] SaXena, R. R. et al., J. Appl. Phys. 51 (1980) 4501.

[6] Zwerdling, S., WANG, K. L. and YeH, Y. C. M., Sixteenth Intersociety Energy Conversion Engineering Conference (1981).

[7] Duchemin, J. P., Bonnet, M., Koeish, F. and HuYghe, D., J. Electrochem. Soc. 126 (1979) 7.

[8] Gave, G., Le Metayer, M., Bouree, J. E., Revue Phys. Appl. (1979).

[9] Hallais, J. P., Acta Electron. 21 (1978) 2.

REVUE PHYSIQUE APPLIQUÉE. - T. 17, № 7, JUILLET 1982
[10] BAss, S. J., J. Crystal Growth 31 (1975) 172.

[11] Seki, Y. and TANNo, K., IIDA, K. and ICHIKI, E., J. Electrochem. Soc. 122 (1975) 8.

[12] Ambridge, T. and Faktor, M. M., Inst. Phys. Conf. Ser. № 24 (1975) 6.

[13] Bass, S. J., J. Crystal Growth 47 (1979) 613.

[14] Bass, S. J. and Oliver, P. E., Inst. Phys. Conf. Ser. No 33 (1977) 1.

[15] Sze, S. M., Physics of Semiconductor Devices.

[16] Manasevit, H. M. and Thorsen, A. C., J. Electrochem. Soc. 119 (1972) 1.

[17] Thorton, Hughes, Sulivay and Wayte, Micro. and Reliability (1966). 